\title{
A new Weibull- $X$ family of distributions: properties, characterizations and applications
}

\author{
Zubair Ahmad ${ }^{1 *}$ (D) M. Elgarhy ${ }^{2}$ and G. G. Hamedani ${ }^{3}$
}

\author{
* Correspondence: \\ z.ferry21@gmail.com \\ 'Department of Statistics, \\ Quaid-i-Azam University 45320, \\ Islamabad 44000, Pakistan \\ Full list of author information is \\ available at the end of the article
}

\begin{abstract}
We propose a new family of univariate distributions generated from the Weibull random variable, called a new Weibull-X family of distributions. Two special sub-models of the proposed family are presented and the shapes of density and hazard functions are investigated. General expressions for some statistical properties are discussed. For the new family, three useful characterizations based on truncated moments are presented. Three different methods to estimate the model parameters are discussed. Monti Carlo simulation study is conducted to evaluate the performances of these estimators. Finally, the importance of the new family is illustrated empirically via two real life applications.
\end{abstract}

Keywords: Weibull distribution, T-X family, Moment, Characterizations, Order statistics, Estimation

\section{Introduction}

In the field of reliability theory, modeling of lifetime data is very crucial. A number of statistical distributions such as Weibull, Pareto, Gompertz, linear failure rate, Rayleigh, Exonential etc., are available for modeling lifetime data. However, in many practical areas, these classical distributions do not provide adequate fit in modeling data, and there is a clear need for the extended version of these classical distributions. In this regard, serious attempts have been made to propose new families of continuous probability distributions that extend the existing well-known distributions by adding additional parameter(s) to the model of the baseline random variable. The well-known family of distributions are: the beta-G by Eugene et al. (2002), Jones (2004), Gamma-G (type-1) due to Zografos and Balakrishnan (2009), Mc-G proposed by Alexander et al. (2012), Log-Gamma-G Type-2 of Amini et al. (2012), Gamma-G (type-2) studied by Risti'c and Balakrishnan (2012), Gamma-G (type-3) of Torabi and Montazeri (2012), Weibull-X family of distributions of Alzaatreh et al. (2013), exponentiated generalized class of Cordeiro et al. (2013), Logistic-G introduced by Torabi and Montazeri (2014), Gamma-X family of Alzaatreh et al. (2014), odd generalized exponential-G of Tahir et al. (2015a, b), type I half-logistic family of Cordeiro et al. (2016), Kumaraswamy Weibull-generated family of Hassan and Elgarhy (2016), new Weibull-G family of Tahir et al. (2016), generalized transmuted-G of Nofal et al. (2017) and a new generalized family of distributions of Ahmad (2018). Let $v(t)$ be the probability density function

(c) The Author(s). 2018 Open Access This article is distributed under the terms of the Creative Commons Attribution 4.0 International License (http://creativecommons.org/licenses/by/4.0/), which permits unrestricted use, distribution, and reproduction in any medium, provided you give appropriate credit to the original author(s) and the source, provide a link to the Creative Commons license, and indicate if changes were made. 
(pdf) of a random variable, say $T$, where $T \in[m, n]$ for $-\infty \leq m<n<\infty$ and let $W[F(x$; $\xi)$ ] be a function of cumulative distribution function (cdf) of a random variable, say $X$, depending on the vector parameter $\xi$ satisfying the conditions given below:

$$
W[F(x ; \xi)] \in[m, n],,
$$

i. $W[F(x ; \xi)]$ is differentiable and monotonically increasing, and

ii. $W[F(x ; \xi)] \rightarrow m$ as $x \rightarrow-\infty$ and $W[F(x ; \xi)] \rightarrow n$ as $x \rightarrow \infty$.

Recently, Alzaatreh et al. (2013), defined the cdf of the T- $X$ family of distributions by

$$
G(x)=\int_{m}^{W[F(x ; \xi)]} v(t) d t, \quad x \in \mathbb{R},
$$

where $W[F(x ; \xi)]$ satisfies the conditions stated above. The pdf corresponding to (1) is

$$
g(x)=\left\{\frac{\partial}{\partial x} W[F(x ; \xi)]\right\} v\{W[F(x ; \xi)]\}, \quad \quad x \in \mathbb{R} .
$$

Using the T- $X$ idea, several new classes of distributions have been introduced in the literature. Table 1 provides some $W[F(x ; \xi)]$ functions for some members of the T-X family.

The main goal of this article is to introduce a new family of continuous distributions, called the new Weibull- $X$ ("NW- $X$ " for short) family. We discuss three special sub-models of this family, capable of modeling with monotonic and non-monotonic hazard rates. For each special sub-model of NW- $X$ family, a real life application is presented.

The rest of this paper is structured as follows: In Section "The New Weibull-X family", we define the NW- $X$ family of distributions. Section "Special sub-Models" offers some special sub-models of this family. Useful expansions for the pdf and cdf of NW- $X$ are provided in section "Useful expansions of the NW-X family". Statistical properties of the NW- $X$ family are investigated in section "Basic mathematical properties". Section "Estimation" provides estimation of the model parameters using maximum likelihood method. Simulation results are reported in section "Simulation Study". Section "Characterizations" contains some useful characterizations of the proposed class. Section "Applications"

Table 1 Some members of the T-X family

\begin{tabular}{lll}
\hline$W[F(x ; \xi)]$ & Range of $X$ & Members of T-X family \\
\hline$F(x ; \xi)$ & {$[0,1]$} & Beta-G (Eugene et al., 2002), Mc-G (Alexander et al., 2012) \\
$-\log [F(x ; \xi)]$ & $(0, \infty)$ & Gamma-G Type-2 (Ristic and Balakrishnan, 2012) \\
$-\log [1-F(x ; \xi)]$ & $(0, \infty)$ & Gamma-G Type-1 (Zografos and Balakrishnan, 2009) \\
$\frac{F(x ; \xi)}{1-F(x ; \xi)}$ & $(0, \infty)$ & Gamma-G Type-3 (Torabi and Montazeri, 2012) \\
$-\log \left[1-F^{a}(x ; \xi)\right]$ & $(0, \infty)$ & Exponentiated T-X (Alzaghal et al., 2013) \\
$\log \left\{\frac{F(x ; \xi)}{1-F(x ; \xi)}\right\}$ & $(-\infty, \infty)$ & Logistic-G (Torabi and Montazeri, 2014) \\
$\log [-\log \{1-F(x ; \xi)\}]$ & $(-\infty, \infty)$ & The Logistic-X Family (Tahir et al., 2015a, b) \\
$\frac{-\log \{1-F(x ; \xi)\}]}{1-F(x ; \xi)}$ & $(0, \infty)$ & New Weibull-X Family (Proposed) \\
\hline
\end{tabular}


provides analysis to real data sets. Finally, section "Concluding Remarks", concludes the article.

\section{The new Weibull-X family}

If $X$ is a Weibull random variable with shape parameter $\alpha>0$ and scale parameter $\beta>0$, then its cdf is given by

$$
V(t ; \alpha, \beta)=1-e^{-\beta t^{\alpha}}, \quad t \geq 0, \alpha, \beta>0 .
$$

Setting $\beta=1$ in (2), we obtain the cdf of the one parameter Weibull random variable given by

$$
V(t ; \alpha)=1-e^{-t^{\alpha}}, \quad t \geq 0, \alpha>0 .
$$

The density function corresponding to (3) is

$$
v(t ; \alpha)=\alpha t^{\alpha-1} e^{-t^{\alpha}}, \quad t \geq 0, \alpha>0 .
$$

If $v(t ; \alpha)$ follows (4) and setting $W[F(x ; \xi)]=\frac{[-\log \{1-F(x ; \xi)\}]}{1-F(x ; \xi)}$ in (1), we define the cdf of the NW-X family by

$$
G(x ; \alpha, \xi)=1-\exp \left[-\left\{\frac{\{-\log (1-F(x ; \xi))\}}{1-F(x ; \xi)}\right\}^{\alpha}\right], \quad \alpha, \xi>0, x \in \mathbb{R},
$$

where, $F(x ; \xi)$ is the cdf of the baseline distribution which depends on the vector parameter $\xi$. We can also write (5) as follows

$$
G(x ; \alpha, \xi)=1-\exp \left[-\left\{\frac{H(x ; \xi)}{1-F(x ; \xi)}\right\}^{\alpha}\right], \quad \alpha, \xi>0, x \in \mathbb{R}
$$

where, $H(x ; \xi)$ is the cumulative hazard rate function (chrf) of the baseline random variable. The density function corresponding to (6) is

$$
g(x ; \alpha, \xi)=\frac{\alpha f(x ; \xi)\{H(x ; \xi)\}^{\alpha-1}\{1+H(x ; \xi)\}}{[1-F(x ; \xi)]^{\alpha+1}} \exp \left[-\left\{\frac{H(x ; \xi)}{1-F(x ; \xi)}\right\}^{\alpha}\right], \quad \alpha, \xi>0, x \in \mathbb{R}
$$

Henceforward, a random variable $X$ with density (7) is denoted by $X \sim N W-X(x ; \alpha, \xi)$. Moreover, we may omit the dependence on the vector $\xi$ of the parameters and simply write $G(x)=G(x ; \xi)$. The survival function (sf), hazard rate function (hrf) and chrf of $X$ are given by (8)-(10), respectively,

$$
\begin{aligned}
& S(x ; \alpha, \xi)=\exp \left[-\left\{\frac{H(x ; \xi)}{1-F(x ; \xi)}\right\}^{\alpha}\right] \\
& h(x ; \alpha, \xi)=\frac{\alpha f(x ; \xi)\{H(x ; \xi)\}^{\alpha-1}\{1+H(x ; \xi)\}}{[1-F(x ; \xi)]^{\alpha+1}}, \\
& H(x ; \alpha, \xi)=\left\{\frac{H(x ; \xi)}{1-F(x ; \xi)}\right\}^{\alpha} .
\end{aligned}
$$

The basic motivations for using the NW- $X$ family in practice are: 
- A prominent method of introducing additional parameter(s) to generate an extended version of the baseline model.

- To improve the characteristics of the traditional distributions.

- To make the kurtosis more flexible compared to the baseline model.

- To generate distributions with symmetric, right-skewed, left-skewed and reversed-J shaped.

- To define special models with all types of hrf.

- To define special models having closed form for cdf, sf as well as hrf.

- To provide consistently better fits than other generated distributions having the same or higher number of parameters.

\section{Special sub-models}

Most of the extended versions of the distributions are introduced for one of the following properties: an alternative model to the existing distribution that has previously been utilized successfully, a statistical model whose empirical distribution is a good fit to the data, and offers greater flexibility. A statistical model having closed forms of cdf, sf as well as hrf and reducing the estimation difficulties. Here, we introduce two special sub-models of NW- $X$ family that can possess at least one of these properties.

\subsection{The NW-Frechet (NW-F) distribution}

The density and cdf of the Frechet random variable are $f(x ; \xi)=\theta \gamma^{\theta} x^{-(\theta+1)} e^{-(\gamma / x)^{\theta}}$, $x>0, \xi>0$, and $F(x ; \xi)=e^{-(\gamma / x)^{\theta}}$, where $\xi=(\gamma, \theta)$. Then the cdf and pdf of the NW-F model are given by

$$
G(x ; \alpha, \xi)=1-\exp \left\{-\left(\frac{-\log \left(1-e^{-(\gamma / x)^{\theta}}\right)}{1-e^{-(\gamma / x)^{\theta}}}\right)^{\alpha}\right\}, \quad x \geq 0, \alpha, \xi>0
$$

and

$$
\begin{aligned}
g(x ; \alpha, \xi)= & \frac{\alpha \theta \gamma^{\theta} x^{-(\theta+1)} e^{-(\gamma / x)^{\theta}}\left\{1-\log \left(1-e^{-(\gamma / x)^{\theta}}\right)\right\}\left\{-\log \left(1-e^{-(\gamma / x)^{\theta}}\right)\right\}^{\alpha-1}}{\left(1-e^{-(\gamma / x)^{\theta}}\right)^{\alpha+1}} \\
& \exp \left\{-\left(\frac{-\log \left(1-e^{-(\gamma / x)^{\theta}}\right)}{1-e^{-(\gamma / x)^{\theta}}}\right)^{\alpha}\right\}, \quad x \geq 0 .
\end{aligned}
$$

Plots of the NW-F density and hrf for selected parameter values are presented in Fig. 1.

\subsection{The NW-Weibull (NW-W) distribution}

Now considering the cdf of the two-parameter Weibull model with shape parameter $\theta>0$ and scale parameter $\gamma>0$, given by $F(x ; \xi)=1-e^{-\gamma x^{\theta}}, x \geq 0, \gamma, \theta>0$, and pdf $f(x$; $\xi)=\gamma \theta x^{\theta-1} e^{-\gamma x^{\theta}}$, where $\xi=(\theta, \gamma)$. Then, the cdf and pdf of the NW-W model are 

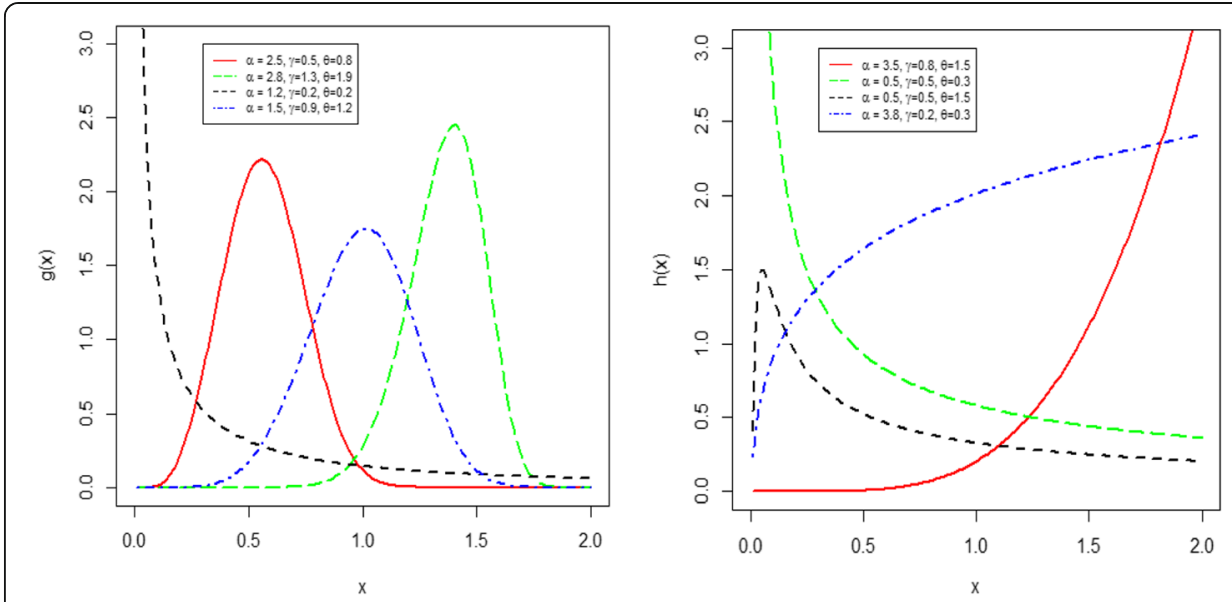

Fig. 1 Different plots for the pdf and hrf of NW-F distribution

$$
G(x ; \alpha, \xi)=1-\exp \left\{-\left(\frac{\gamma x^{\theta}}{e^{-\gamma x^{\theta}}}\right)^{\alpha}\right\}, \quad x \geq 0, \alpha, \xi>0
$$

and

$$
g(x ; \alpha, \xi)=\alpha \gamma^{\alpha} \theta x^{\alpha \theta-1} e^{\alpha \gamma x^{\theta}}\left\{1+\gamma x^{\theta}\right\} \exp \left\{-\left(\frac{\gamma x^{\theta}}{e^{-\gamma x^{\theta}}}\right)^{\alpha}\right\}, \quad x \geq 0
$$

respectively.

Figure 2 displays plots of the NW-W pdf and hrf for selected parameter values.

\section{Useful expansions of the NW-X family}

In this section, we provide useful expansions for the pdf and cdf of the NW- $X$ family. Using the series
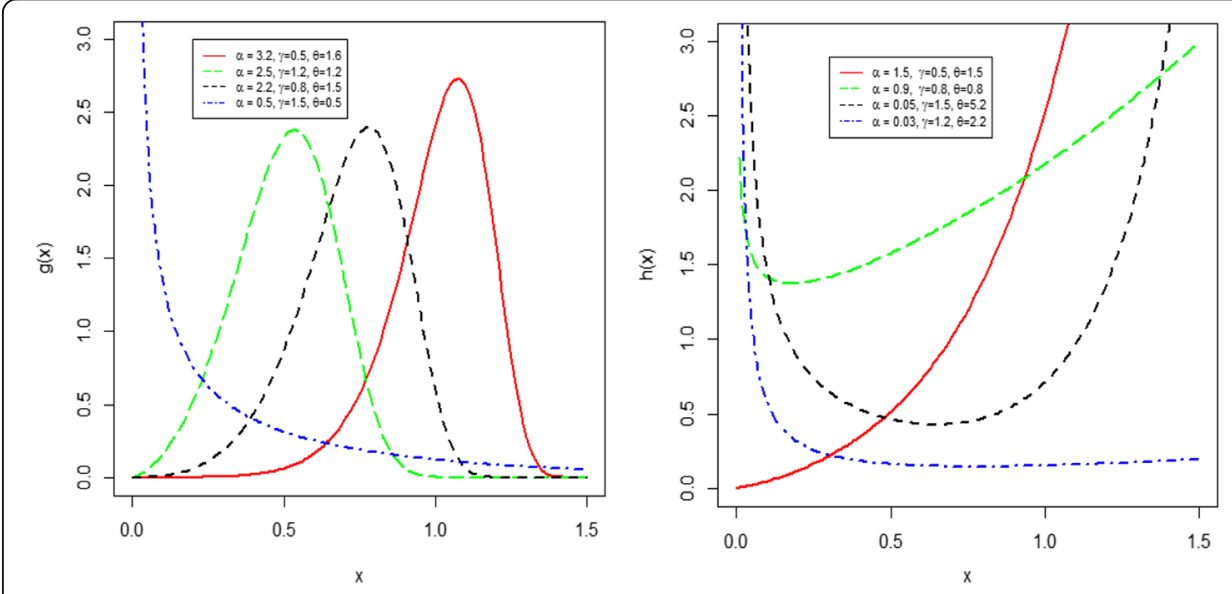

Fig. 2 Different plots for the pdf and hrf of NW-W distribution 


$$
e^{-x}=\sum_{i=0}^{\infty} \frac{(-1)^{i}}{i} x^{i}
$$

from (7), we obtain

$$
g(x ; \alpha, \xi)=\sum_{i=0}^{\infty} \frac{(-1)^{i}}{i !} \frac{\alpha f(x ; \xi)\left[\{H(x ; \xi)\}^{\alpha(i+1)}+\{H(x ; \xi)\}^{\alpha(i+1)-1}\right]}{[1-F(x ; \xi)]^{\alpha(i+1)+1}} .
$$

Hence, the pdf (16) can be written as

$$
g(x ; \alpha, \xi)=\sum_{i=0}^{\infty} \sum_{j=0}^{\infty} \eta_{i, j}\left(\tau_{i, j}+\tau_{i, j}^{\prime}\right)
$$

where $\eta_{i, j}=\alpha \frac{(-1)^{i+j}}{i !}\left(\begin{array}{c}\alpha(i+1) \\ j\end{array}\right), \tau_{i, j}=f(x ; \xi)\{H(x ; \xi)\}^{\alpha(i+1)}\{F(x ; \xi)\}^{j}$ and $\tau_{i, j}^{\prime}=f(x ; \xi)$ $\{H(x ; \xi)\}^{\alpha(i+1)-1}\{F(x ; \xi)\}^{j}$.

Furthermore, we provide an expansion for $\{G(x ; \alpha, \xi)\}^{s}$, where $s$ is an integer. Using the binomial theorem, we have

$$
\{G(x ; \alpha, \xi)\}^{s}=\sum_{k=0}^{\infty}\left(\begin{array}{l}
s \\
k
\end{array}\right) \exp \left[-k\left\{\frac{H(x ; \xi)}{1-F(x ; \xi)}\right\}^{\alpha}\right] .
$$

Using the series (15) in (18), we are arrive at

$$
\{G(x ; \alpha, \xi)\}^{s}=\sum_{k, l=0}^{\infty}(-1)^{l}\left(\begin{array}{c}
s \\
k
\end{array}\right) k^{l} \frac{\{H(x ; \xi)\}^{\alpha l}}{\{1-F(x ; \xi)\}^{\alpha l}} .
$$

Finally,

$$
\{G(x ; \alpha, \xi)\}^{s}=\sum_{k, l, m=0}^{\infty}(-1)^{l+m}\left(\begin{array}{c}
s \\
k
\end{array}\right)\left(\begin{array}{c}
\alpha l \\
m
\end{array}\right) k^{l}\{H(x ; \xi)\}^{\alpha l}\{F(x ; \xi)\}^{m} .
$$

\section{Basic mathematical properties}

In this section, we provide some basic properties of the NW- $X$ family.

\subsection{Moments}

Let $X$ follows the NW- $X$ family with density (17), then its $r^{\text {th }}$ moment is

$$
\mu_{r}^{\prime}=\sum_{i=0}^{\infty} \sum_{j=0}^{\infty} \eta_{i, j} \int_{-\infty}^{\infty} x^{r}\left(\tau_{i, j}+\tau_{i, j}^{\prime}\right) d x, \mu_{r}^{\prime}=\sum_{i=0}^{\infty} \sum_{j=0}^{\infty} \eta_{i, j}\left(\Lambda_{r, i, j}+\Delta_{r, i, j}^{\prime}\right),
$$

where.

$$
\Lambda_{r, i, j}=\int_{-\infty}^{\infty} x^{r} \tau_{i, j} d x \text { and } \Lambda_{r, i, j}^{\prime}=\int_{-\infty}^{\infty} x^{r} \tau_{i, j}^{\prime} d x \text {. }
$$

Furthermore, the moment generating function (mgf) of $X$ is given by

$$
M_{x}(t)=\sum_{i=0}^{\infty} \sum_{j=0}^{\infty} \sum_{r=0}^{\infty} \frac{t^{r}}{r !} \eta_{i, j}\left(\Lambda_{r, i, j}+\Delta_{r, i, j}^{\prime}\right)
$$




\subsection{Probability weighted moments}

The probability weighted moment (pwm) of NW- $X$ random variable is

$$
M_{r, s}(x)=\int_{-\infty}^{\infty} x^{s} g(x ; \alpha, \xi)(G(x ; \alpha, \xi))^{s} d x .
$$

Using (17) and (19) in (22), we have

$$
M_{r, s}(x)=\int_{-\infty}^{\infty} x^{s} \sum_{i, j=0}^{\infty} \sum_{k, l, m=0}^{\infty} \kappa_{i, j, k, l, m}\left(\rho_{i, j, m}+\rho_{i, j, m}^{l}\right) d x,
$$

Where $\quad \kappa_{i, j, k, l, m}=\eta_{i, j} \sum_{k, l, m=0}^{\infty}(-1)^{l+m}\left(\begin{array}{c}s \\ k\end{array}\right)\left(\begin{array}{c}\alpha l \\ m\end{array}\right) k^{l}, \rho_{i, j, m}=f(x ; \xi)\{H(x ; \xi)\}^{\alpha(i+l+1)}$ $\{F(x ; \xi)\}^{m+j}$ and $\rho_{i, j, m}^{l}=f(x ; \xi)\{H(x ; \xi)\}^{\alpha(i+l+1)-1}\{F(x ; \xi)\}^{m+j}$.

Then,

$$
M_{r, s}(x)=\sum_{i, j=0}^{\infty} \sum_{k, l, m=0}^{\infty} \kappa_{i, j, k, l, m}\left(\psi_{i, j, m, s}+\psi_{i, j, m, s}^{\prime}\right),
$$

where, $\psi_{i, j, m, s}=\int_{-\infty}^{\infty} x^{s} \rho_{i, j, m} d x$ and $\psi_{i, j, m, s}^{\prime}=\int_{-\infty}^{\infty} x^{s} \rho_{i, j, m}^{\prime} d x$.

\subsection{Quantile function}

The quantile function of $X$ with cdf (5) is given by

$$
(-\log (1-u))^{1 / \alpha}-F(x ; \xi)(-\log (1-u))^{1 / \alpha}+\log [1-F(x ; \xi)]=0,
$$

where $u$ is the uniform random number in $(0,1)$. The expression (25) does not have a closed form, therefore, computer software can be used to obtain a closed form solution of the quantile function.

\subsection{Order statistics}

Let $X_{1}, X_{2}, \cdots, X_{k}$ be independent and identically distributed (i.i.d) random variables from NW- $X$ distribution with parameters $\alpha$ and $\xi$. Let $X_{1: k}, X_{2: k}, \cdots, X_{k: k}$ be the corresponding order statistics. Then, the density of $X_{r: k}$ for $(r=1,2, \ldots, k)$ is given by

$$
g_{r: k}(x)=\frac{g(x ; \alpha, \xi)}{B(r, k-r+1)} \sum_{v=0}^{k-r}\left(\begin{array}{c}
k-r \\
v
\end{array}\right)(-1)^{v}[G(x ; \alpha, \xi)]^{v+r-1}
$$

where, $B(.$, .) represents the beta function. Using (17) and (19) in (26) and replacing $s$ with $v+r$-1, we have

$$
g_{r: k}(x)=\frac{\sum_{i, j=0}^{\infty} \sum_{k, l, m=0}^{\infty} \sum_{v=0}^{k-r} \boldsymbol{r}_{i, j, k, l, m, v}}{B(r, k-r+1)}\left(\rho_{i, j, m}+\rho_{i, j, m}^{\prime}\right),
$$

where, $\Upsilon_{i, j, k, l, m, v}=(-1)^{v}\left(\begin{array}{c}k-r \\ v\end{array}\right) \kappa_{i, j, k, l, m}$. 


\section{Estimation}

Here, we describe three methods of estimation of the unknown parameters of the NW-X family. The methods are: ordinary least square (OLS) estimation, percentile based estimation and maximum likelihood estimation. The performance of these estimation methods are studied through Monte Carlo simulation.

\subsection{Ordinary Least Square estimation}

Let $x_{1}, x_{2}, \ldots, x_{k}$ be the observed ordered values from the sample $X_{1}, X_{2}, \ldots, X_{k}$ from NW- $X$ family. Then, the expectation of the empirical cdf is defined as

$$
E\left[G\left(x_{(i)} ; \alpha, \xi\right)\right]=\frac{i}{k+1} ; \quad i=1,2, \ldots, k
$$

The OLS estimates $\hat{\alpha}$ and $\hat{\xi}$ of $\alpha$ and $\xi$ can be determined by maximizing

$$
Z(\alpha, \xi)=\sum_{i=1}^{k}\left(G\left(x_{(i)} ; \alpha, \xi\right)-\frac{i}{k+1}\right)^{2}
$$

Consequently, ordinary least square estimators (OLSEs) $\hat{\alpha}$ and $\hat{\xi}$ of $\alpha$ and $\xi$ can be obtained as the solution of the following equations

$$
\begin{aligned}
& \frac{\partial Z(\alpha, \xi)}{\partial \alpha}=\sum_{i=1}^{k}\left(G\left(x_{(i)} ; \alpha, \xi\right)-\frac{i}{k+1}\right) \frac{\partial G\left(x_{(i)} ; \alpha, \xi\right)}{\partial \alpha}=0 \\
& \frac{\partial Z(\alpha, \xi)}{\partial \xi}=\sum_{i=1}^{k}\left(G\left(x_{(i)} ; \alpha, \xi\right)-\frac{i}{k+1}\right) \frac{\partial G\left(x_{(i)} ; \alpha, \xi\right)}{\partial \xi}=0 .
\end{aligned}
$$

The OLS estimates of $(\alpha, \xi)$ are the simultaneous solutions of $\frac{\partial Z(\alpha, \xi)}{\partial \alpha}=0$ and $\frac{\partial Z(\alpha, \xi)}{\partial \xi}=0$.

\subsection{Percentile estimation}

In this subsection, we estimate the unknown parameters by using the percentile estimation method. Let $p_{i}=\frac{1}{k+1}$ be an estimate of $G\left(x_{(i)} ; \alpha, \xi\right)$, then the percentile estimators of $(\alpha, \xi)$ can be obtained by maximizing the function

$$
\phi=\sum_{i=1}^{k}\left\{\ln \left(p_{i}\right)-\log G\left(x_{(i)} ; \alpha, \xi\right)\right\}^{2} .
$$

Henceforth, the percentile estimates $\hat{\alpha}$ and $\hat{\xi}$ of $\alpha$ and $\xi$ are obtained as the simultaneous solution of the expressions given in (33) and (34), below

$$
\begin{aligned}
& \frac{\partial \phi}{\partial \alpha}=-2 \sum_{i=1}^{k} \frac{\left\{\ln \left(p_{i}\right)-\log G\left(x_{(i)} ; \alpha, \xi\right)\right\} \partial G\left(x_{(i)} ; \alpha, \xi\right) / \partial \alpha}{G\left(x_{(i)} ; \alpha, \xi\right)}=0, \\
& \frac{\partial \phi}{\partial \xi}=-2 \sum_{i=1}^{k} \frac{\left\{\ln \left(p_{i}\right)-\log G\left(x_{(i)} ; \alpha, \xi\right)\right\} \partial G\left(x_{(i)} ; \alpha, \xi\right) / \partial \xi}{G\left(x_{(i)} ; \alpha, \xi\right)}=0 .
\end{aligned}
$$

Setting $\frac{\partial \phi}{\partial \alpha}$ and $\frac{\partial \phi}{\partial \xi}$ equal to zero and solving numerically these expressions simultaneously yield the percentile estimators (PEs) of $(\alpha, \xi)$. 


\subsection{Maximum likelihood estimation}

In this subsection, we determine the maximum likelihood estimates of the parameters of the NW- $X$ family. Let $x_{1}, x_{2}, \cdots, x_{k}$ be the observed values from the NW- $X$ distribution with parameters $\alpha$ and $\xi$. The total log- likelihood function corresponding to (7) is given by

$$
\begin{gathered}
\log L\left(x_{i} ; \alpha, \xi\right)=k \log \alpha+\sum_{i=1}^{k} \log f\left(x_{i} ; \xi\right)+\sum_{i=1}^{k} \log \left\{\left(H\left(x_{i} ; \xi\right)\right)^{\alpha-1}+\left(H\left(x_{i} ; \xi\right)\right)^{\alpha}\right\} \\
-\alpha \sum_{i=1}^{k} \frac{H\left(x_{i} ; \xi\right)}{1-F\left(x_{i} ; \xi\right)}-(\alpha+1) \sum_{i=1}^{k} \log \left[1-F\left(x_{i} ; \xi\right)\right] .
\end{gathered}
$$

The partial derivatives of (35) are

$$
\begin{gathered}
\frac{\partial}{\partial \alpha} \log L\left(x_{i} ; \alpha, \xi\right)=\frac{k}{\alpha}+\sum_{i=1}^{k} \frac{\left(\log H\left(x_{i} ; \xi\right)\right)\left(H\left(x_{i} ; \xi\right)\right)^{\alpha-1}+\left(\log H\left(x_{i} ; \xi\right)\right)\left(H\left(x_{i} ; \xi\right)\right)^{\alpha}}{\left\{\left(H\left(x_{i} ; \xi\right)\right)^{\alpha-1}+\left(H\left(x_{i} ; \xi\right)\right)^{\alpha}\right\}} \\
-\sum_{i=1}^{k} \frac{H\left(x_{i} ; \xi\right)}{1-F\left(x_{i} ; \xi\right)}-\sum_{i=1}^{k} \log \left[1-F\left(x_{i} ; \xi\right)\right] \\
\frac{\partial}{\partial \xi} \log L\left(x_{i} ; \alpha, \xi\right)=\sum_{i=1}^{k} \frac{\partial f\left(x_{i} ; \xi\right) / \partial \xi}{f\left(x_{i} ; \xi\right)}+\sum_{i=1}^{k} \frac{\left(H\left(x_{i} ; \xi\right)\right)^{\alpha-2}\left((\alpha-1)+\alpha H\left(x_{i} ; \xi\right)\right)}{\left\{\left(H\left(x_{i} ; \xi\right)\right)^{\alpha-1}+\left(H\left(x_{i} ; \xi\right)\right)^{\alpha}\right\}}+(\alpha+1) \sum_{i=1}^{k} \frac{\partial F\left(x_{i} ; \xi\right) / \partial \xi}{\left[1-F\left(x_{i} ; \xi\right)\right]} \\
-\alpha \sum_{i=1}^{k} \frac{\left(1-F\left(x_{i} ; \xi\right)\right) h\left(x_{i} ; \xi\right)+H\left(x_{i} ; \xi\right) f\left(x_{i} ; \xi\right)}{1-F\left(x_{i} ; \xi\right)}
\end{gathered}
$$

Setting $\frac{\partial}{\partial \alpha} \log L\left(x_{i} ; \alpha, \xi\right)$ and $\frac{\partial}{\partial \xi} \log L\left(x_{i} ; \alpha, \xi\right)$ equal to zero and solving numerically these expressions simultaneously yield the maximum likelihood estimators (MLEs) of $(\alpha, \xi)$.

\section{Simulation study}

In this section, we carry out simulation study for NW-W model. The process is described below:

i. Random samples of sizes $n=30,100$ are generated from NW-W model and parameters have been estimated via ordinary least square, percentile and maximum likelihood methods.

ii. 1000 repetitions are made to calculate the bias and mean square error (MSE) of

these estimators.
iii. Formulas used for calculating bias and MSE are given by $\operatorname{Bias}(\hat{\alpha})=\frac{1}{1000} \sum_{i=1}^{1000}\left(\hat{\alpha}_{i}-\alpha\right)$
and $\operatorname{MSE}(\hat{\alpha})=\frac{1}{1000} \sum_{i=1}^{1000}\left(\hat{\alpha}_{i}-\alpha\right)^{2}$, respectively. 
iv. Step (iii) is also repeated for the other parameters $(\theta, \gamma)$.

The results are provided in Tables 2 and 3.

\section{Characterizations}

This section deals with the characterizations of the NW-X distribution in different directions: (i) based on the ratio of two truncated moments; (ii) in terms of the hazard function and (iii) based on the conditional expectation of certain function of the random variable. Note that (i) can be employed also when the cdf does not have a closed form. We would also like to mention that due to the nature of NW-X distribution, our characterizations may be the only possible ones. We present our characterizations (i)-(iii) in three subsections.

\subsection{Characterizations based on two truncated moments}

This subsection is concerned with the characterizations of NW-X distribution based on the ratio of two truncated moments. Our first characterization employs a theorem due to Glänzel (1987); see Theorem 1 of Appendix. The result, however, holds also when the interval $\mathrm{H}$ is not closed, since the condition of the Theorem is on the interior of $\mathrm{H}$.

Proposition 8.1. Let $X: \Omega \rightarrow \mathbb{R}$ be a continuous random variable and let $q_{1}(x) \equiv 1$ and $q_{2}(x)=\exp \left[-\left\{\frac{-\log (1-F(x ; \xi))}{1-F(x ; \xi)}\right\}^{\alpha}\right]$ for $x \in \mathbb{R}$. The random variable $X$ has pdf (7) if and only if the function $\eta(x)$ defined in Theorem 1 is of the form

$$
\eta(x)=\frac{1}{2} \exp \left[-\left\{\frac{-\log (1-F(x ; \xi))}{1-F(x ; \xi)}\right\}^{\alpha}\right], \quad \quad x \in \mathbb{R} .
$$

Proof. Suppose the random variable $X$ has pdf (7), then

$$
(1-F(x)) E\left(q_{1}(X) \mid X \geq x\right)=\exp \left[-\left\{\frac{-\log (1-F(x ; \xi))}{1-F(x ; \xi)}\right\}^{\alpha}\right], \quad x \in \mathbb{R},
$$

and

$$
(1-F(x)) E\left(q_{2}(X) \mid X \geq x\right)=\frac{1}{2} \exp \left[-\left\{\frac{-\log (1-F(x ; \xi))}{1-F(x ; \xi)}\right\}^{\alpha}\right], \quad x \in \mathbb{R} .
$$

\begin{tabular}{|c|c|c|c|c|c|c|}
\hline \multicolumn{7}{|c|}{ Set 1: $a=0.5, \theta=0.2, y=1.5$} \\
\hline \multirow[t]{2}{*}{$n$} & \multicolumn{2}{|l|}{ MLES } & \multicolumn{2}{|l|}{ OLSES } & \multicolumn{2}{|l|}{ PES } \\
\hline & Estimates & MSES & Estimates & MSES & Estimates & MSEs \\
\hline \multirow[t]{3}{*}{30} & 0.243115 & 0.006933 & 0.500000 & $2.62247^{*} 10^{\wedge}-13$ & 0.499999 & $1.20077^{*} 10^{\wedge}-12$ \\
\hline & 0.038949 & 0.002646 & 0.200000 & $3.69762^{*} 10^{\wedge}-14$ & 0.200000 & $1.69355^{*} 10^{\wedge-13}$ \\
\hline & 2.215720 & 0.011471 & 1.500000 & $2.42796^{*} 10^{\wedge}-12$ & 1.500000 & $9.23321^{*} 10^{\wedge}-12$ \\
\hline \multirow[t]{3}{*}{100} & 0.243066 & 0.006983 & 0.500000 & $2.41978^{*} 10 \wedge-13$ & 0.499999 & $1.17444^{*} 10^{\wedge}-12$ \\
\hline & 0.038940 & 0.002669 & 0.200000 & $3.42612^{*} 10^{\wedge}-14$ & 0.200000 & $1.6606^{*} 10^{\wedge}-13$ \\
\hline & 2.215470 & 0.011554 & 1.500000 & $2.23827^{*} 10^{\wedge}-12$ & 1.500000 & $9.02771 * 10^{\wedge}-12$ \\
\hline
\end{tabular}

Further,

Table 2 Average values of estimates and the corresponding MSES 
Table 3 Average values of estimates and the corresponding MSES

\begin{tabular}{|c|c|c|c|c|c|c|}
\hline \multicolumn{7}{|c|}{ Set $2: a=0.8, \theta=0.2, y=1.5$} \\
\hline \multirow[t]{2}{*}{$n$} & \multicolumn{2}{|l|}{ MLES } & \multicolumn{2}{|l|}{ OLSES } & \multicolumn{2}{|l|}{ PES } \\
\hline & Estimates & MSES & Estimates & MSES & Estimates & MSES \\
\hline \multirow[t]{3}{*}{30} & 0.625068 & 0.009387 & 0.800000 & $1.48037^{*} 10^{\wedge}-15$ & 0.800000 & $2.08848^{*} 10^{\wedge}-14$ \\
\hline & 0.038624 & 0.003470 & 0.200000 & $2.97589^{*} 10^{\wedge}-17$ & 0.200000 & $4.14824^{*} 10 \wedge-16$ \\
\hline & 2.206900 & 0.014437 & 1.500000 & $6.85963^{*} 10^{\wedge}-15$ & 1.500000 & $7.49312^{*} 10^{\wedge}-14$ \\
\hline \multirow[t]{3}{*}{100} & 0.625035 & 0.009409 & 0.800000 & $1.06613^{*} 10^{\wedge}-15$ & 0.800000 & $1.15878^{*} 10^{\wedge}-14$ \\
\hline & 0.038620 & 0.003481 & 0.200000 & $2.14695^{*} 10^{\wedge}-17$ & 0.200000 & $2.33517^{*} 10^{\wedge}-16$ \\
\hline & 2.206810 & 0.014468 & 1.500000 & $4.93807^{*} 10 \wedge-15$ & 1.500000 & $4.14635^{*} 10 \wedge-14$ \\
\hline
\end{tabular}

$$
\eta(x) q_{1}(x)-q_{2}(x)=\frac{1}{2} \exp \left[-\left\{\frac{-\log (1-F(x ; \xi))}{1-F(x ; \xi)}\right\}^{\alpha}\right]<0, \quad x \in \mathbb{R} .
$$

Conversely, if $\eta(x)$ is of the above form, then

$$
\frac{\eta^{\prime}(x) q_{1}(x)}{\eta(x) q_{1}(x)-q_{2}(x)}=\frac{\alpha f(x ; \xi)\{-\log (1-F(x ; \xi))\}^{\alpha-1}[1+\{-\log (1-F(x ; \xi))\}]}{(1-F(x ; \xi))^{\alpha+1}}, \quad x \in \mathbb{R},
$$

Now, according to Theorem $1, X$ has density (7).

Corollary 8.1. Let $\mathrm{X}: \Omega \rightarrow \mathbb{R}$ be a continuous random variable and let $q_{1}(x)$ be as in Proposition 8.1. The random variable $X$ has pdf (7) if and only if there exist functions $q_{2}(x) \operatorname{and} \eta(x)$ defined in Theorem 1 satisfying the following differential equation

$$
\frac{\eta^{\prime}(x) q_{1}(x)}{\eta(x) q_{1}(x)-q_{2}(x)}=\frac{\alpha f(x ; \xi)\{-\log (1-F(x ; \xi))\}^{\alpha-1}[1+\{-\log (1-F(x ; \xi))\}]}{(1-F(x ; \xi))^{\alpha+1}}, \quad x \in \mathbb{R},
$$

Corollary 8.2. The general solution of the differential equation in Corollary 8.1 is

$$
\eta(x)=\exp \left[-\left\{\frac{-\log (1-F(x ; \xi))}{1-F(x ; \xi)}\right\}^{\alpha}\right]\left[\begin{array}{c}
-\int \frac{\alpha f(x ; \xi)\{-\log (1-F(x ; \xi))\}^{\alpha-1}[1+\{-\log (1-F(x ; \xi))\}]}{(1-F(x ; \xi))^{\alpha+1}} \\
\times \exp \left[-\left\{\frac{-\log (1-F(x ; \xi))}{1-F(x ; \xi)}\right\}^{\alpha}\right]\left(q_{1}(x)\right)^{-1} q_{2}(x) d x+D
\end{array}\right],
$$

where $D$ is a constant. We like to point out that one set of functions satisfying the above differential equation is given in Proposition 8.1 with $D=1$. Clearly, there are other triplets $\left(q_{1}(x), q_{2}(x), \eta(x)\right)$ which satisfy conditions of Theorem 1 .

\subsection{Characterization in terms of hazard function}

The hazard function $h_{G}(x)$ of a twice differentiable distribution function, $G(x)$, satisfies the following first order differential equation

Table 4 Ordinary least square estimates of the fitted models for the first data set

\begin{tabular}{llll}
\hline Dist. & $\hat{a}$ & $\hat{\gamma}$ & $\hat{\theta}$ \\
\hline NW-F & 3.354 & 3.3270 & 0.173 \\
F & & 3.251 & 0.752 \\
EF & 1.475 & 1.940 & 0.7510 \\
\hline
\end{tabular}


Table 5 Goodness-of-fit measures of the fitted models for the first data set

\begin{tabular}{llllllll}
\hline Dist. & KS & CM & AD & AIC & BIC & CIAC & HQIC \\
\hline NW-F & 0.070 & 0.095 & 0.583 & 831.52 & 840.08 & 831.72 & 835.00 \\
F & 0.140 & 0.744 & 4.545 & 892.00 & 897.70 & 892.09 & 894.31 \\
EF & 0.169 & 0.798 & 4.643 & 894.00 & 902.55 & 894.19 & 897.47 \\
\hline
\end{tabular}

$$
\frac{g^{\prime}(x)}{g(x)}=\frac{h_{G}^{\prime}(x)}{h_{G}(x)}-h_{G}(x)
$$

It should be mentioned that for many univariate continuous distributions, the above equation is the only differential equation available in terms of the hazard function. In this subsection we present a non-trivial characterization of NW-W distribution in terms of the hazard function.

Proposition 8.2. Let $\mathrm{X}: \Omega \rightarrow \mathbb{R}$ be a continuous random variable. The random variable $X$ has pdf (7) if and only if its hazard function $h_{G}(x)$ satisfies the following differential equation

$$
\begin{aligned}
h_{G}^{\prime}(x) & =\frac{(\alpha-1) f(x ; \xi)}{\{-\log (1-F(x ; \xi))\}(1-F(x ; \xi))} h_{G}(x) \\
& =\alpha\{-\log (1-F(x ; \xi))\}^{\alpha-1} \frac{d}{d x}\left[\frac{f(x ; \xi)[1+\{-\log (1-F(x ; \xi))\}]}{(1-F(x ; \xi))^{\alpha+1}}\right] \quad x \in \mathbb{R} .
\end{aligned}
$$

Proof. If $X$ has density (7), then clearly the above differential equation holds. Now, if the differential equation holds, then

$$
\frac{d}{d x}\left[\{-\log (1-F(x ; \xi))\}^{1-\alpha} h_{F}(x)\right]=\alpha \frac{d}{d x}\left[\frac{f(x ; \xi)[1+\{-\log (1-F(x ; \xi))\}]}{(1-F(x ; \xi))^{\alpha+1}}\right], \quad x \in \mathbb{R}
$$

from which we arrive at the hazard function of (9).

\subsection{Characterization based on the conditional expectation of certain function of the} random variable

In this subsection we employ a single function $\psi$ of $X$ and characterize the distribution of $X$ in terms of the truncated moment of $\psi(x)$. The following proposition has already
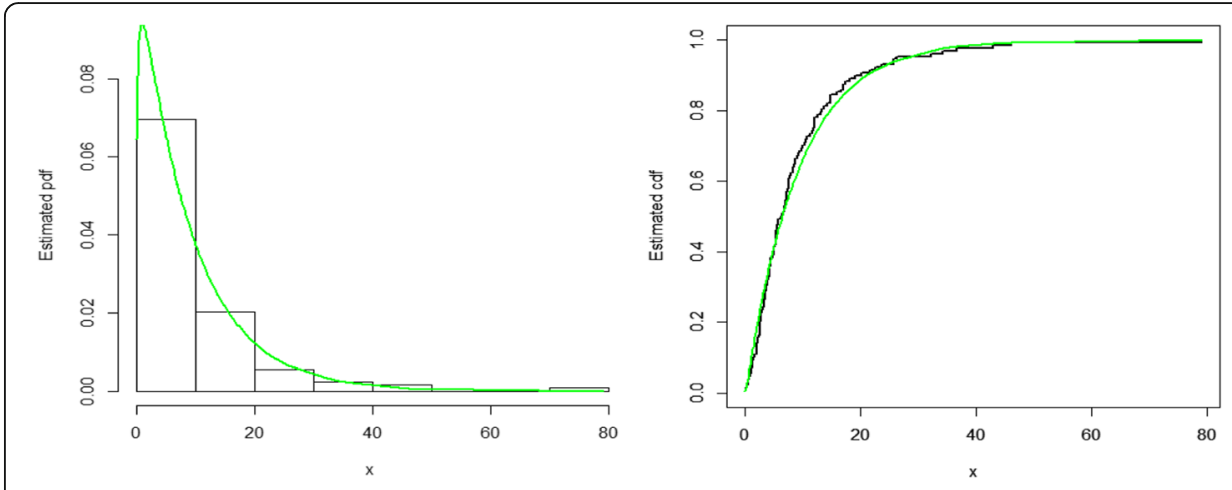

Fig. 3 Estimated pdf and cdf of NW-F distribution using data 1 

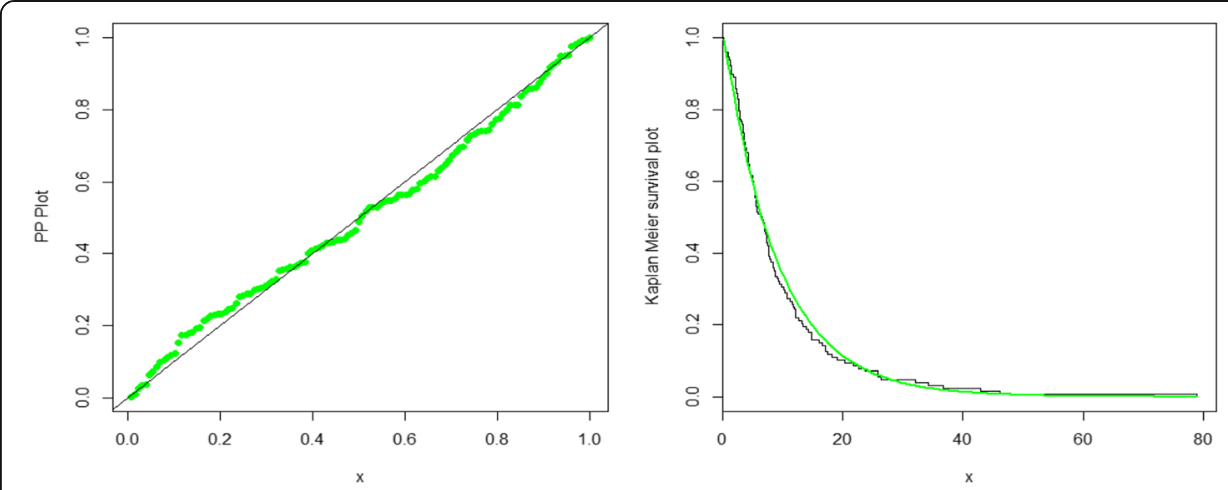

Fig. 4 Kaplan Meier and PP-plots of NW-F distribution using data 1

appeared in Hamedani's previous work (2013), so we will just state it here which can be used to characterize the NW- $X$ distribution.

Proposition 8.3. Let $\mathrm{X}: \Omega \rightarrow(e, f)$ be a continuous random variable with cdf $F$. Let $\psi(x)$ be a differentiable function on $(e, f)$ with $\lim _{x \rightarrow e^{+}} \psi(x)$. Then for $\delta \neq 1$,

$$
E[\psi(X) \mid X \geq x]=\delta \psi(x), \quad x \in(e, f),
$$

if and only if

$$
\psi(x)=\{1-F(x ; \alpha, \xi)\}^{\frac{1}{\delta}-1}, \quad x \in(e, f)
$$

Remark 8.1. For $(e, f)=\mathbb{R}, \psi(x)=\exp \left[-\left\{\frac{-\log (1-F(x ; \xi))}{1-F(x ; \xi)}\right\}^{\alpha}\right]$ and $\delta=\frac{1}{2}$, Proposition 8.3 provides a characterization of $\mathrm{NW}-X$ distribution.

\section{Applications}

In the following section, we provide two applications of the $\mathrm{NW}-X$ family using real data for illustrative purposes. These applications show the flexibility and usefulness of the new generator. For these data sets, we compare the fits of the proposed distribution to other well-known distributions. In order to compare the models, we consider the following analytical measures: Akaike information criterion (AIC), Consistent Akaike Information Criterion (CAIC), Bayesian information criterion (BIC), Hannan-Quinn information criterion (HQIC), Kolmogorov-Smirnov (KS) test statistic, Cramer-von Mises (CM) statistic and Anderson-Darling (AD) test statistic.

Data 1 The first data set obtained from Lee and Wang (2003) representing the remission times (in months) of a random sample of 128 bladder cancer patients. We applied

Table 6 Maximum likelihood estimates of the fitted distributions using the second data set

\begin{tabular}{llllll}
\hline Dist. & $\hat{a}$ & $\hat{\gamma}$ & $\hat{\theta}$ & $\hat{a}$ & $\hat{b}$ \\
\hline NW-W & 5.355 & 0.222 & 0.600 & & \\
APTW & 4.958 & 0.003 & 3.931 & \\
FWE & 4.073 & 5.961 & 3.832 & \\
MW & 1.034 & 0.008 & 3.873 & & \\
EW & 4.867 & 0.007 & 3.620 & 1.663 & 2.301 \\
Ku-W & & 0.009 & 3.657 & \\
\hline
\end{tabular}


Table 7 Goodness-of-fit measures of the fitted distributions for the second data set

\begin{tabular}{llllllll}
\hline Dist. & KS & CM & AD & AIC & BIC & CIAC & HQIC \\
\hline NW-W & 0.048 & 0.060 & 0.389 & 343.16 & 351.49 & 343.37 & 346.54 \\
APTW & 0.144 & 0.082 & 0.513 & 347.21 & 355.55 & 347.42 & 350.60 \\
FWE & 0.109 & 0.126 & 0.731 & 349.95 & 358.29 & 350.16 & 353.34 \\
MW & 0.079 & 0.111 & 0.675 & 349.43 & 357.77 & 349.64 & 352.82 \\
EW & 0.082 & 0.168 & 1.045 & 347.72 & 356.05 & 347.92 & 351.10 \\
Ku-W & 0.059 & 0.121 & 0.757 & 348.10 & 359.21 & 348.45 & 352.61 \\
\hline
\end{tabular}

the NW-F distribution to this data set in competition with Frechet $(F)$ and exponentiated Frechet (EF) distributions. The cdf of the EF distribution proposed by Nadarajah Kotz (2003) is given by

$$
G(x ; \alpha, \xi)=1-\left[1-\exp \left\{-\left(\frac{\gamma}{x}\right)^{\theta}\right\}\right]^{\alpha}, \quad x, \alpha, \theta, \gamma>0 .
$$

The maximum likelihood estimates of the model parameters and analytical results are provided in Tables 4 and 5; Figs. 3 and 4, respectively.

Data 2 The second data set representing strength of the Alumina (Al2O3) material taken from the website: http://www.ceramics.nist.gov/srd/summary/ftmain.htm. This data set can also be found in Nadarajah and Kotz (2008). We applied another sub-model of the proposed family NW-W distribution to this data set in competition with five other well-known competing distributions. The distribution functions of the competing models are

- Exponentiated Weibull (EW) of Mudholkar and Srivastava (1993)

$$
G(x)=\left(1-e^{-\gamma x^{\theta}}\right)^{\alpha}, \quad x, \alpha, \theta, \gamma>0 .
$$

- Modified Weibull (MW) of Lai et al. (2003)

$$
G(x)=1-e^{-\gamma x^{\theta} e^{\alpha x}}, \quad x, \alpha, \theta, \gamma>0 .
$$

- Kumaraswamy Weibull (Ku-W) of Cordeiro et al. (2010)
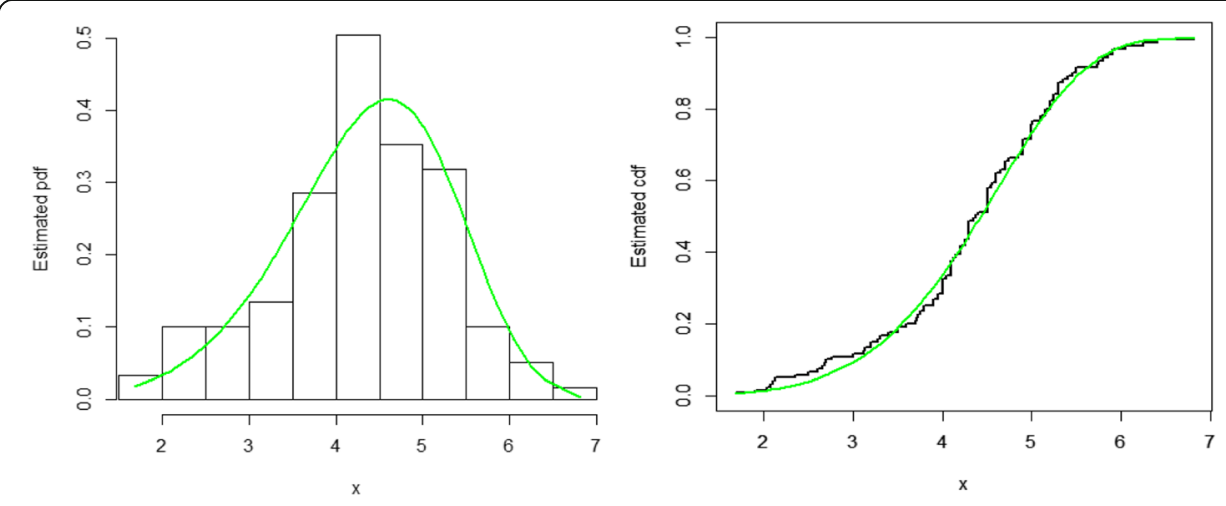

Fig. 5 Estimated pdf and cdf of NW-W distribution using data 2 

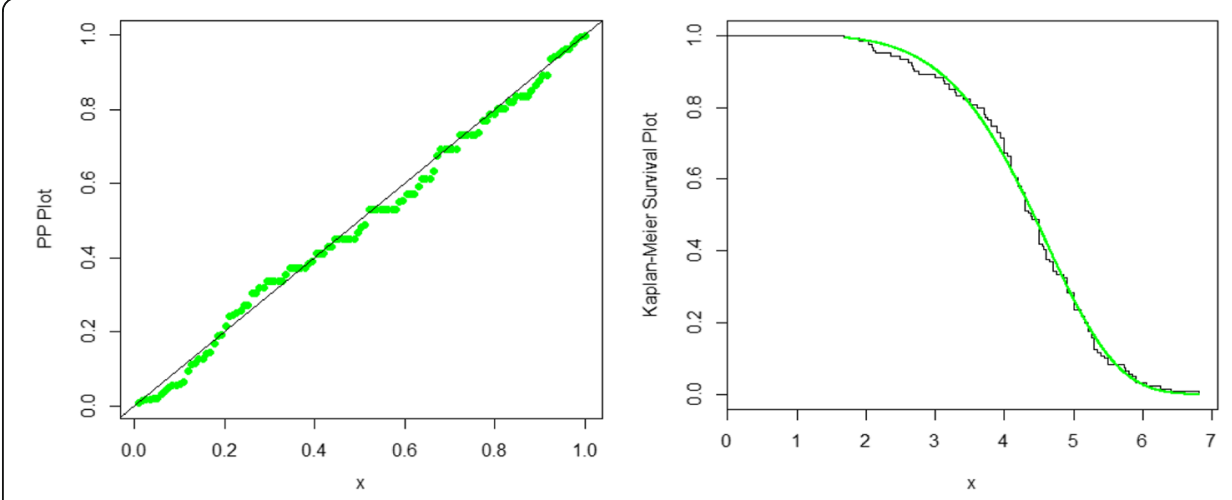

Fig. 6 Kaplan Meier and PP-plots of NW-W distribution using data 2

$$
G(x)=1-\left(1-\left(1-e^{-\gamma x^{\theta}}\right)^{a}\right)^{b}, \quad x, a, b, \theta, \gamma>0
$$

- Alpha power transformed Weibull (APTW) of Dey et al. (2017)

$$
G(x)=\frac{\alpha^{\left(1-e^{-\gamma x^{\theta}}\right)}-1}{\alpha-1}, \quad \quad x, \alpha, \theta, \gamma>0, \alpha \neq 1 .
$$

- Flexible Weibull extended (FWE) of Ahmad and Hussain (2017)

$$
G(x)=1-\exp \left(-e^{\alpha x^{2}-\frac{\gamma}{x^{\theta}}}\right), \quad x, \alpha, \theta, \gamma>0 .
$$

The maximum likelihood estimates of the model parameters and analytical results are provided in Tables 6 and 7; Figs. 5 and 6, respectively.

\section{Concluding remarks}

We have introduced a new family of distributions, called a new Weibull- $X$ family. Two special sub-models of this family are discussed. The densities of two special models can be left-skewed, right-skewed, symmetrical, reverse J-shaped and can have increasing, decreasing, unimodal or most importantly bathtub shaped failure rates. The parameters are estimated using three different methods, namely, ordinary least square, percentile estimation and maximum likelihood. A simulation study is presented to evaluate the performances of the model parameters estimators. Some mathematical properties of the new family are also derived. Two real applications of the proposed family are provided, and reveal better fits to data than other well-known distributions.

\section{Appendix}

Theorem 1 . Let $(\Omega, \quad, P)$ be a given probability space and let $H=[a ; b]$ be an interval for some $a<b$ ( $a=-\infty ; b=\infty$ might as well be allowed). Let $X: \Omega \rightarrow H$ be a continuous random variable with the distribution function $\mathrm{F}$ and let $q_{1}(x)$ and $q_{2}(x)$ be two real functions defined on $\mathrm{H}$ such that

$$
E\left(q_{2}(X) \mid X \geq x\right)=E\left(q_{1}(X) \mid X \geq x\right) \eta(x), \quad x \in H,
$$

is defined with some real function $\eta(x)$. Assume that $q_{1}(x), q_{2}(x) \in C^{1}(H), \eta(x) \in C^{2}(x)$ 
and $\mathrm{F}$ is twice continuously differentiable and strictly monotone function on the set $H$. Finally, assume that the equation $\xi q_{1}(x)=q_{2}(x)$ has no real solution in the interior of $H$. Then $F$ is uniquely determined by the functions $q_{1}(x), q_{2}(x)$ and $\eta(x)$ particularly

$$
F(x)=\int_{a}^{x} C\left|\frac{\eta^{\prime}(u)}{\eta(u) q_{1}(u)-q_{2}(u)}\right| \exp (-s(u)) d u,
$$

where the function $s(u)$ is a solution of the differential equation $s^{\prime}(u)=\frac{\eta^{\prime}(u) q_{1}(u)}{\eta(u) q_{1}(u)-q_{2}(u)}$ and $\mathrm{C}$ is the normalization constant, such that $\int_{H} d F=1$..

We like to mention that this kind of characterization based on the ratio of truncated moments is stable in the sense of weak convergence (see, Glänzel (1990)), in particular, let us assume that there is a sequence $\left\{X_{n}\right\}$ of random variables with distribution functions $\left\{F_{n}\right\}$ such that the functions $q_{1 n}, q_{2 n}$ and $\eta_{n}(n \in \mathbb{N})$ satisfy the conditions of Theorem 1 and let $q_{1 n} \rightarrow q_{1}, q_{2 n} \rightarrow q_{2}$ for some continuously differentiable real functions $q_{1}(x)$ and $q_{2}(x)$. Let, finally, $X$ be a random variable with distribution $F(x)$. Under the condition that $q_{1 n}(x)$ and $q_{2 n}(x)$ are uniformly integrable and the family $\left\{F_{n}\right\}$ is relatively compact, the sequence $X_{n}$ converges to $X$ in distribution if and only if $\eta_{n}$ converges to $\eta$, where

$$
\eta(x)=\frac{E\left(q_{2}(X) \mid X \geq x\right)}{E\left(q_{1}(X) \mid X \geq x\right)} .
$$

This stability theorem makes sure that the convergence of distribution functions is reflected by corresponding convergence of the functions $q_{1}(x), q_{2}(x)$ and $\eta(x)$ respectively. It guarantees, for instance, the 'convergence' of characterization of the Wald distribution to that of the Levy-Smirnov distribution if $\alpha \rightarrow \infty$. A further consequence of the stability property of Theorem 1 is the application of this theorem to special tasks in statistical practice such as the estimation of the parameters of discrete distributions. For such purpose, the functions $q_{1}(x), q_{2}(x)$ and, specially, $\eta(x)$ should be as simple as possible. Since the function triplet is not uniquely determined it is often possible to choose $\eta(x)$ as a linear function. Therefore, it is worth analyzing some special cases which helps to find new characterizations reflecting the relationship between individual continuous univariate distributions and appropriate in other areas of statistics.

\begin{abstract}
Abbreviations
AD: Anderson-Darling; AIC: Akaike information criterion; APTW: Alpha power transformed Weibull; BIC: Bayesian information criterion; CAIC: Consistent Akaike Information Criterion; cdf: Cumulative distribution function; chrf: Cumulative hazard rate function; CM: Cramer-von Mises; EF: Exponentiated Frechet; EW: Exponentiated Weibull; F: Frechet; FWE: Flexible Weibull extended; HQIC: Hannan-Quinn information criterion; hrf: Hazard rate function; i.i.d: Independent and identically distributed; KS: Kolmogorov-Smirnov; Ku-W: Kumaraswamy Weibull; mgf: Moment generating function; MLEs: Maximum likelihood estimators; MSE: Mean square error; MW: Modified Weibull; NW-F: NWFrechet; NW-W: NW-Weibull; NW-X: New Weibull-X; OLS: Ordinary least square; OLSEs: Ordinary least square estimators; pdf: Probability density function; PEs: Percentile estimators; pwm: Probability weighted moment; sf: Survival function
\end{abstract}

Acknowledgements

The authors are grateful to the Editor-in-Chief, the Associate Editor and anonymous referees for many of their valuable comments and suggestions which lead to this improved version of the manuscript. 


\section{Availability of data and materials}

The idea of T-X family of distributions proposed by Alzaatreh et al. (2013) has been used.

Data 1 The first data set taken from Lee and Wang (2003) are as follows: $0.08,2.09,3.48,4.87,6.94,8.66,13.11,23.63$, $0.20,2.23,3.52,4.98,6.97,9.02,13.29,0.40,2.26,3.57,5.06,7.09,9.22,13.80,25.74,0.50,2.46,3.64,5.09,7.26,9.47,14.24$ $25.82,0.51,2.54,3.70,5.17,7.28,9.74,14.76,26.31,0.81,2.62,3.82,5.32,7.32,10.06,14.77,32.15,2.64,3.88,5.32,7.39$, $10.34,14.83,34.26,0.90,2.69,4.18,5.34,7.59,10.66,15.96,36.66,1.05,2.69,4.23,5.41,7.62,10.75,16.62,43.01,1.19,2.75$, $4.26,5.41,7.63,17.12,46.12,1.26,2.83,4.33,5.49,7.66,11.25,17.14,79.05,1.35,2.87,5.62,7.87,11.64,17.36,1.40,3.02$ $4.34,5.71,7.93,11.79,18.10,1.46,4.40,5.85,8.26,11.98,19.13,1.76,3.25,4.50,6.25,8.37,12.02,2.02,3.31,4.51,6.54,8.53$, $12.03,20.28,2.02,3.36,6.76,12.07,21.73,2.07,3.36,6.93,8.65,12.63,22.69$

Data 2 The second data set taken from the website: http://www.ceramics.nist.gov/srd/summary/ftmain.htm are as follows: 5.5, 5, 4.9, 6.4, 5.1, 5.2, 5.2, 5, 4.7, 4, 4.5, 4.2, 4.1, 4.56, 5.01, 4.7, 3.13, 3.12, 2.68, 2.77, 2.7, 2.36, 4.38, 5.73, 4.35, 6.81, $1.91,2.66,2.61,1.68,2.04,2.08,2.13,3.8,3.73,3.71,3.28,3.9,4,3.8,4.1,3.9,4.05,4,3.95,4,4.5,4.5,4.2,4.55,4.65,4.1$, 4.25, $4.3,4.5,4.7,5.15,4.3,4.5,4.9,5,5.35,5.15,5.25,5.8,5.85,5.9,5.75,6.25,6.05,5.9,3.6,4.1,4.5,5.3,4.85,5.3,5.45,5.1,5.3$, $5.2,5.3,5.25,4.75,4.5,4.2,4,4.15,4.25,4.3,3.75,3.95,3.51,4.13,5.4,5,2.1,4.6,3.2,2.5,4.1,3.5,3.2,3.3,4.6,4.3,4.3,4.5$, $5.5,4.6,4.9,4.3,3,3.4,3.7,4.4,4.9,4.9,5$

\section{Authors' contributions}

The authors, ZA, ME and GGH with the consultation of each other carried out this research and drafted the manuscript together. All authors read and approved the final version of the manuscript.

\section{Competing interests}

The authors declare that they have no competing interests.

\section{Publisher's Note}

Springer Nature remains neutral with regard to jurisdictional claims in published maps and institutional affiliations.

\section{Author details}

'Department of Statistics, Quaid-i-Azam University 45320, Islamabad 44000, Pakistan. ${ }^{2}$ Vice Presidency for Graduate Studies and Scientific Research, University of Jeddah, Jeddah, Kingdom of Saudi Arabia. ${ }^{3}$ Department of Mathematics, Statistics and Computer Science, Marquette University, Milwaukee WI 53201-1881, USA.

Received: 19 June 2018 Accepted: 17 October 2018

Published online: 03 November 2018

\section{References}

Ahmad, Z: A new generalized class of distributions: properties and estimation based on type-I censored samples. Annals of Data Science. (2018). https://doi.org/10.1007/s40745-018-0160-5

Ahmad, Z., \& Hussain, Z. (2017). Flexible Weibull extended distribution. MAYFEB Journal of Materials Science. 2, 5-18 (2017)

Alexander, C., Cordeiro, G.M., Ortega, E.M.M., Sarabia, J.M.: Generalized beta-generated distributions. Computational Statistics and Data Analysis. 56, 1880-1897 (2012)

Alzaatreh, A., Famoye, F., Lee, C.: A new method for generating families of continuous distributions. Metron. 71, 63-79 (2013)

Alzaatreh, A., Famoye, F., Lee, C.: The gamma-normal distribution: properties and applications. Computational Statistics and Data Analysis. 69, 67-80 (2014)

Alzaghal, A., Lee, C., Famoye, F.: Exponentiated T-X family of distributions with some applications. International Journal of Probability and Statistics. 2, 31-49 (2013)

Amini, M., Mir Mostafaee, S.M.T.K., Ahmadi, J.: Log-gamma-generated families of distributions. Statistics, iFirst. (2012). https:// doi.org/10.1008/02331888.2012.748775

Cordeiro, G.M., Alizadeh, M., Diniz Marinho, P.R.: The type I half-logistic family of distributions. Journal of Statistical Computation and Simulation. 86(4), 707-728 (2016)

Cordeiro, G.M., Ortega, E.M., Nadarajah, S.: The Kumaraswamy Weibull distribution with application to failure data. Journal of the Franklin Institute. 347(8), 1399-1429 (2010)

Cordeiro, G.M., Ortega, E.M.M., Cunha, D.C.C.: The exponentiated generalized class of distributions. Journal of Data Science. 11 $1-27(2013)$

Dey, S., Sharma, V.K., Mesfioui, M.: A new extension of Weibull distribution with application to lifetime data. Annals of Data Science. 4(1), 31-61 (2017)

Eugene, N., Lee, C., Famoye, F.: Beta-normal distribution and its applications. Communications in Statistics Theory and Methods. 31, 497-512 (2002)

Glänzel, W. (1987). A characterization theorem based on truncated moments and its application to some distribution families, mathematical statistics and probability theory (bad Tatzmannsdorf, 1986), Vol. B, Reidel, Dordrecht, 7584

Glänzel, W.: Some consequences of a characterization theorem based on truncated moments, statistics: a journal of theoretical and. Applied Statistics. 21(4), 613-618 (1990)

Hamedani, G.G. (2013). On certain generalized gamma convolution distributions II, technical report no. 484, MSCS, Marquette University

Hassan, A.S., Elgarhy, M.: Kumaraswamy Weibull-generated family of distributions with applications. Advances and Applications in Statistics. 48, 205-239 (2016)

Jones, M.C.: Families of distributions arising from the distributions of order statistics. Test. 13, 1-43 (2004)

Lai, C.D., Xie, M., Murthy, D.N.P.: A modified Weibull distribution. IEEE Transactions on reliability. 52(1), 33-37 (2003)

Lee, E.T., Wang, J.W.: Statistical Methods for Survival Data Analysis, 3rd edn. Wiley, New York (2003)

Mudholkar, G.S., Srivastava, D.K.: Exponentiated Weibull family for analyzing bathtub failure-rate data. IEEE transactions on reliability. 42(2), 299-302 (1993) 
Nadarajah, S., Kotz, S. (2003). The Exponentiated Fr'echet Distribution. InterStat. Available online at http://interstat.statjournals. net/YEAR/2003/abstracts/0312001.php

Nadarajah, S., Kotz, S.: Strength modeling using Weibull distributions. Journal of mechanical science and technology. 22(7), 1247-1254 (2008)

Nofal, Z.M., Afify, A.Z., Yousof, H.M., Cordeiro, G.M.: The generalized transmuted-G family of distributions. Communication in Statistics-Theory Methods. 46, 4119-4136 (2017)

Risti'c, M.M., Balakrishnan, N.: The gamma-exponentiated exponential distribution. Journal of Statistical Computation and Simulation. 82, 1191-1206 (2012)

Tahir, M.H., Cordeiro, G.M., Alizadeh, M., Mansoor, M., Zubair, M., Hamedani, G.G.: The odd generalized exponential family of distributions with applications. Journal of Statistical Distributions and Applications. 2(1), 1-28 (2015a)

Tahir, M. H., Cordeiro, G. M., Alzaatreh, A., Mansoor, M., Zubair, M. (2015b). The Logistic-X Family of Distributions and its Applications

Tahir, M.H., Zubair, M., Mansoor, M., Cordeiro, G.M., Alizadeh, M., Hamedani, G.G.: A new Weibull-G family of distributions. Hacettepe Journal of Mathematics and Statistics. 45(2), 629-647 (2016)

Torabi, H., Montazari, N.H.: The gamma-uniform distribution and its application. Kybernetika. 48, 16-30 (2012)

Torabi, H., Montazari, N.H.: The logistic-uniform distribution and its application. Communications in Statistics-Simulation and Computation. 43, 2551-2569 (2014)

Zografos, K., Balakrishnan, N.: On families of beta- and generalized gamma-generated distributions and associated inference. Statistical Methodology. 6, 344-362 (2009)

Submit your manuscript to a SpringerOpen ${ }^{\circ}$ journal and benefit from:

- Convenient online submission

- Rigorous peer review

- Open access: articles freely available online

- High visibility within the field

- Retaining the copyright to your article

Submit your next manuscript at $>$ springeropen.com 